\title{
SPR Imaging at Shorter Wavelengths by Using Cytop Inserted Sensor Chips
}

\author{
Yasunori Iribe, Atsuya Yamamoto, Masayasu Suzuki \\ University of Toyama, Toyama, Toyama 930-8555, Japan \\ suzukimy@eng.u-toyama.ac.jp
}

\begin{abstract}
:
Long range surface plasmon (LRSP) technique was used in order to achieve high SPR sensitivity even at shorter wavelength visible light source $(<550 \mathrm{~nm})$. Insertion of Cytop layer, which has similar refractive index with water, beneath gold film caused improvement of SPR curve sharpness and negative shift of SPR angle. By inserting Cytop layer, SPR imaging with gold sensor film was realized even at $505 \mathrm{~nm}$, which is useful wavelength for surface plasmon enhanced fluorometry (SPEF). SPR sensitivity increased over 15 -fold at $590 \mathrm{~nm}$ by inserting Cytop layer. The present result might contribute to improvement of SPR image resolution and SPEF measurement.
\end{abstract}

Key words: SPR, long range surface plasmon, Cytop, surface plasmon enhanced fluorometry

\section{Introduction}

Two dimensional surface plasmon resonance (SPR) imaging technologies enable real-time monitoring of SPR images on the surface of DNA or protein array. Generally, to obtain higher sensitivity, longer wavelength visible light is used for SPR sensor light source. But resolution of SPR image increases by the use of shorter wavelength visible light as light source [1]. Surface plasmon enhanced fluorometry (SPEF) is also promising technique for near field fluorescence measurement. Many useful fluorescence dyes are excited with light shorter than 550nm. We have been studying on SPR measurement with shorter wavelength light source, and developing new SPR sensor chips for this purpose. Gold is the most popular SPR sensor chip, but SPR measurement with shorter than $600 \mathrm{~nm}$ light was impossible. We focused on long range SPR technique. LRSPS are surface electromagnetic waves that can be created on thin metallic films imbedded between two identical dielectrics. By inserting amorphous fluorine resin Cytop, which has similar refractive index to water $\left(n_{D}=1.34\right)$, beneath metal film, LRSPR sensor can be realized [2]. In this study, we evaluated performance of SPR imaging using Cytopinserted gold sensor chip.

\section{Materials and Methods}

\section{D-SPR Imaging Sensor}

The 2D-SPR imaging sensor we used is based on a Kretschmann configuration (2DSPR-04A, NTT Advanced Technology). The incident beam is transverse magnetically polarized and reflected light is imaged onto the cooled CCD camera via a microscopic long distance lens (1 7X).High power LEDs (505, 590, 660 and 850nm, DC2100, Thorlabs) were used for light sources.

\section{Sensor chip}

SF6 $\left(n_{D}=1.805\right)$ was used as a sensor chip substrate. Cytop ( $n_{D}=1.34$, CTL-809M, Asahi Chemicals) was spin-coated on SF6 substrate. After drying (room temp. for 60min and 160C for $60 \mathrm{~min})$. Gold layer was coated on it by ion sputtering.

\section{Results and Discussion}

\section{Optimization of Cytop and gold thickness}

Optimal thickness of Cytop and gold layers were determined from SPR curves obtained at 4 kinds of wavelengths as shown in Table 1.

Table 1.Optimal preparation conditions for LRSPR sensor chips at various wavelengths

\begin{tabular}{|c|c||c|}
\hline $\begin{array}{c}\text { Light source } \\
\lambda\end{array}$ & cytop & gold \\
\hline $505 \mathrm{~nm}$ & $50 \%$ Cytop,2000rpm & $30 \mathrm{~nm}$ \\
\hline $590 \mathrm{~nm}$ & $80 \%$ Cytop,4000rpm & $40 \mathrm{~nm}$ \\
\hline $660 \mathrm{~nm}$ & $90 \%$ Cytop,4000rpm & $40 \mathrm{~nm}$ \\
\hline $850 \mathrm{~nm}$ & $100 \%$ Cytop,2500rpm & $40 \mathrm{~nm}$ \\
\hline
\end{tabular}




\section{Effect of Cytop insertion on SPR curves}

SPR curves of a Cytop inserted LRSPR sensor was compared with those of a conventional SPR sensor. Figure 1 shows a result at $660 \mathrm{~nm}$. SPR curves became sharper and HWHM (Half Width at Half Maximum) of adsorption peak was decreased for $0.7^{\circ}$. Shift of SPR angle was $3.1^{\circ}$. Fig. 2 shows the result at $505 \mathrm{~nm}$. SPR curves became sharper and large negative shift of SPR angle could be observed by inserting Cytop. SPR measurement with gold sensor chip became possible by inserting Cytop. This might be strong advantage for SPEF, because various useful fluorescence dye can be used.

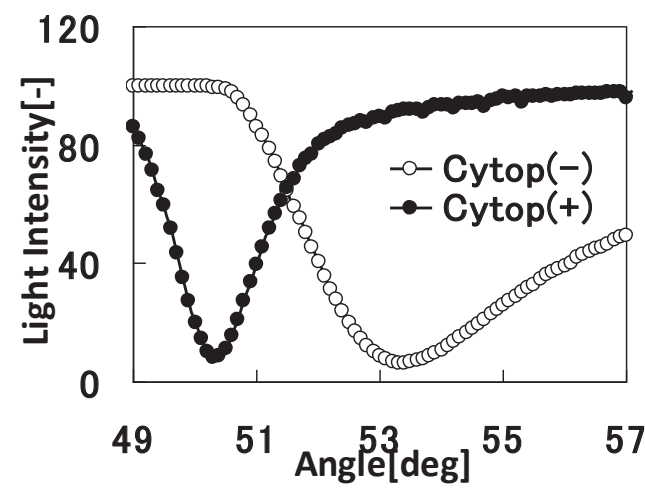

Fig. 1 SPR curves at 660nm light

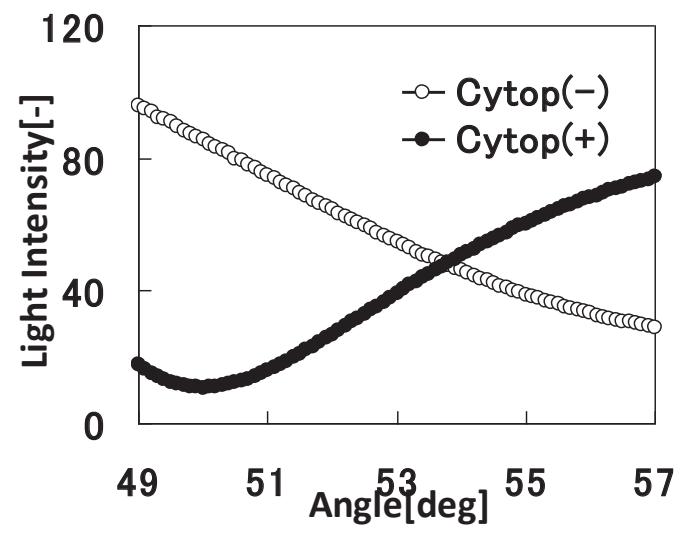

Fig. 2 SPR corves at $505 \mathrm{~nm}$

\section{Effect of Cytop layer insertion on SPR sensor sensitivity}

Sensitivity of the SPR sensor using a Cytop inserted sensor chip was investigated by using $50 \mathrm{~g} / \mathrm{l}$ glucose solution (Fig.3). SPR angle shift became smaller than conventional SPR sensor by using a Cytop inserted sensor chip. But light intensity change increased more than 15 -fold at $590 \mathrm{~nm}$ by inserting Cytop layer. Decrease of HWHM, index of sharpness of SPR curve, might contribute to this effect.

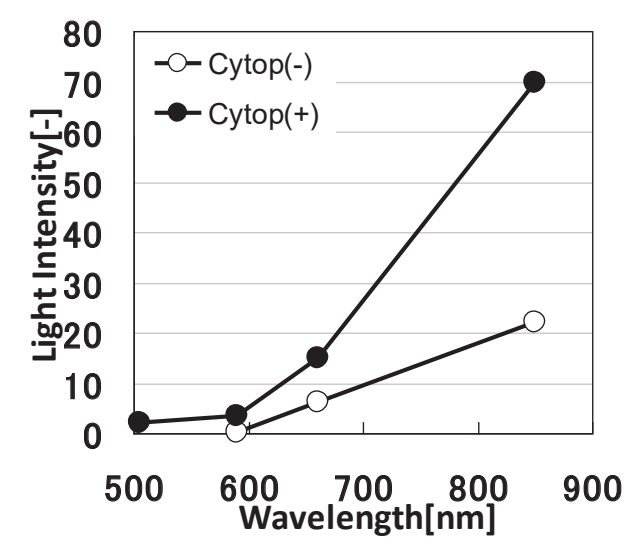

Fig. 3 Responses to $50 \mathrm{~g} / \mathrm{l}$ glucose

Cytop layer insertion and penetration depth Effect of Cytop insertion on penetration depth was investigated, and obtained results were summarized in Table 2. Penetration depth, $L_{p}$, was calculated by the following equation [3].

$L_{p}=\frac{\lambda}{2 \pi \sqrt{n_{p}{ }^{2} \sin ^{2}\left(\theta_{\text {res }}\right)-n_{s}{ }^{2}}}$

Where $\lambda$ is wavelength of light source, $n_{p}$ and $n_{s}$ are refractive index of SF6 substrate and water, $\theta$ res is SPR angle.

Penetration depth was increased 1.6 -fold by Cytop insertion.

Table 2. Penetration depth of LRSPR sensor

\begin{tabular}{|c|c|c|}
\hline & Calculated Lp & Measured Lp \\
\hline Cytop(-) & 356 & $351 \pm 39$ \\
\hline Cytop(+) & 520 & $558 \pm 58$ \\
\hline Cytop(+)/(-) & 1.46 & 1.59 \\
\hline
\end{tabular}

\section{References}

[1] M. Suzuki, Y.Iribe, T.Tobita, Handbook of Biosensors and Biochips (eds. R.S.Marks et al., ), Wiley (2007)

[2] A.W.Wark et al., Long-range surface Plasmon resonance imaging for bioaffinity sensors, Anal.Chem., 77, 3904-3907 (2005); doi: 10.1021/ac050402v

[3] R.Mejard et al., tunable and robust long range surface Plasmon resonance for biosensing applications, Optical Materials, 35, 2507-2513 (2013); doi: 10.1016/j.optmat.2013.07.011 